Research Paper

\title{
Modulation of Salmonella Tumor-Colonization and Intratumoral Anti-angiogenesis by Triptolide and Its Mechanism
}

\author{
Jianxiang Chen ${ }^{1 *}$, Yiting Qiao ${ }^{*}$, Bo Tang ${ }^{2}$, Guo Chen ${ }^{1}$, Xiufeng Liu ${ }^{1}$, Bingya Yang ${ }^{1}$, Jing Wei ${ }^{1}$, Xiangyu \\ Zhang ${ }^{1}$, Xiawei Cheng ${ }^{1}$, Pan Du ${ }^{1}$, Wenhui Jiang ${ }^{1}$, Qingang $\mathrm{Hu}^{1}$ and Zi-Chun Hua ${ }^{1}{ }^{\circledR}$ \\ 1. School of Life Sciences and Collaborative Innovation Center of Chemistry for Life Sciences, School of Stomatology, Affiliated Stomatological Hospital, \\ Nanjing University, Nanjing 210023, People's Republic of China; \\ 2. Changzhou High-Tech Research Institute of Nanjing University and Jiangsu TargetPharma Laboratories Inc., Changzhou 213164, P. R. China. \\ * These authors contributed equally to the work. \\ $\triangle$ Corresponding author: Zi-Chun Hua, School of Life Science, Nanjing University, Nanjing 210023, China. Tel: 0086 -25-83324605 Fax: 0086-25-83324605 E-mail: \\ huazc@nju.edu.cn \\ (c) Ivyspring International Publisher. This is an open access article distributed under the terms of the Creative Commons Attribution (CC BY-NC) license \\ (https://creativecommons.org/licenses/by-nc/4.0/). See http://ivyspring.com/terms for full terms and conditions.
}

Received: 2016.12.19; Accepted: 2017.03.22; Published: 2017.06.01

\begin{abstract}
The weakened tumour colonization of attenuated Salmonella has severely hampered its clinical development. In this study, we investigated whether an anti-inflammation and antiangiogenesis compound triptolide could improve the efficacy of VNP20009, a highly attenuated Salmonella strain, against mice melanoma. By comparing the effects of conventional VNP20009 monotherapy and a combination therapy that uses both triptolide and VNP20009, we found that triptolide significantly improved the tumour colonization of VNP20009 by reducing the number of infiltrated neutrophils in the melanoma, which led to a larger necrotic area in the melanoma. Moreover, the combination therapy suppressed tumour angiogenesis by reducing the expression of VEGF in a synergistic manner, retarding the growth of the melanoma. Our study revealed that triptolide could significantly enhance the antitumour effect of VNP20009 by modulating tumour angiogenesis and the host immune response, providing a new understanding of the strategy to improve Salmonella-mediated tumour therapy.
\end{abstract}

Key words: Salmonella-mediated tumor therapy, melanoma, neutrophils, angiogenesis.

\section{Introduction}

Salmonella enterica serovar Typhimurium (S. Typhimurium), an oncolytic bacterium and tumour-specific vehicle for therapeutic genes [1, 2], has been genetically attenuated to obtain a safer antitumour effect with a higher specificity and a lower systematic toxicity by deleting genes involved in the synthesis of some key factors, such as adenine and lipopolysaccharide [3]. Attenuated S. Typhimurium specifically colonizes solid tumours, which are more hypoxic than healthy tissue, inducing the necrosis and apoptosis of cancer cells through invasive bacterial infection or by expressing cytotoxic genes [3, 4]. Even though attenuated $S$. Typhimurium achieved remarkable antitumour effects in animal models, little clinical success has been obtained in human cancer patients $[5,6]$, which is a result that is probably related to the high complexity of the human immune system and the overattenuation of $S$. Typhimurium, both of which have led to poor tumour colonization and weak biological effects.

The host immune response is one of the key factors that modulate Salmonella's antitumour effects [7-9]. Salmonella triggers a cascade of cellular signalling events, including NFKB signalling, immediately upon its entry into the body, leading to the release of cytokines and chemokines, which 
recruit polymorphonuclear cells [10]. These inflammatory cells play different roles in Salmonella-colonized tumours $[8,9,11]$. Neutrophils directly cause the elimination of bacteria from the body $[8,11]$, while $\mathrm{CD}^{+} \mathrm{T}$ cells contribute to the tumour growth suppression effect of Salmonella [12, 13]. Therefore, reducing the number of neutrophils and/or increasing the percentage of $\mathrm{CD}^{+} \mathrm{T}$ cells should improve the tumour suppression efficacy of $S$. Typhimurium.

The anaerobic tumour microenvironment and the chemokines secreted by tumour cells and/or adjacent tissues strongly attract Salmonella to colonize the necrotic and hypoxic regions in solid tumours [4, 14]. This resident Salmonella can cause the lysis of tumour cells and the fragmentation of the nuclei, resulting in larger necrotic and hypoxic regions in tumours [15]. Moreover, this resident Salmonella also suppresses intratumoural neoangiogenesis by inducing the apoptosis and necrosis of endothelial cells and by reducing the VEGF level and percentage of CD31 positive cells in tumours, resulting in a more hypoxic tumour microenvironment $[4,16]$. This positive feedback loop between the hypoxic tumour microenvironment and Salmonella colonization theoretically provides a way to improve the tumour suppression efficacy of Salmonella by suppressing intratumoural neoangiogenesis with angiogenesis inhibitors, which should create an ideal anaerobic microenvironment in the tumour that supplements the conventional monotherapy that uses Salmonella. The combination of an antiangiogenesis drug and Salmonella has achieved a significantly improved efficacy in a pancreatic cancer patient-derived orthotopic xenograft (PDOX) model [17].

Triptolide is a potent anti-inflammatory and antiangiogenesis diterpenoid triepoxide compound purified from Tripterygium wilfordii Hook. F. (Celastraceae), which has been used to treat autoimmune and inflammatory diseases in China for centuries [18]. Triptolide has been shown to suppress the growth and metastasis of many kinds of solid tumours, including melanoma [19-21]. As a broad-spectrum anticancer and antiangiogenesis drug [22], the combination therapy of triptolide and standard chemotherapy drugs, such as cisplatin, showed dramatic synergistic effects [23]. However, whether the combination of triptolide and Salmonella has a similar synergistic effect has not been studied.

In this study, we used the highly attenuated $S$. Typhimurium VNP20009 variant, which has been proved to be highly effective in experimental models and highly safe in initial clinical trials $[5,6]$ but has failed to show any convincing therapeutic effects in later trials $[5,24]$. Unlike in mouse models, in which
VNP20009 actively colonizes a tumour at low doses, only extremely high doses or the continuous administration of VNP20009 has led to tumour colonization in humans $[5,6]$. Therefore, we hypothesize that the therapeutic effect of VNP20009 can be optimized by combining it with triptolide, which modulates the host immune response and intratumoural neoangiogenesis.

In this study, we demonstrated that triptolide significantly improved the tumour colonization of VNP20009 by suppressing the anti-Salmonella host immune response, especially the infiltration of neutrophils. Triptolide also inhibited intratumoural angiogenesis to create a more hypoxic tumour microenvironment that favoured bacterial proliferation. Our findings enriched the understanding of key factors regulating the tumour colonization of Salmonella and improved the tumour-targeting Salmonella therapy for further clinical developments.

\section{Materials and Methods}

\section{Cells, bacteria and colony formation assays}

B16F10 melanoma cells and Jurkat cells were obtained from the American Type Culture Collection (ATCC, Rockville, MD) and grown as previously described [16]. Lipid A-modified $\left(m s b B^{-}\right)$auxotrophic (purI-) S. Typhimurium strain VNP20009 (ATCC) was grown at $37^{\circ} \mathrm{C}$ to the mid-logarithmic phase in LB broth (without sodium chloride). For colony formation assays, the number of colony forming units (CFUs) was determined as previously described [4, 16].

\section{Animal studies}

Six to seven week-old female C57BL/6 mice, which were purchased from the Laboratory Animal Center, Yangzhou University, Yangzhou, Jiangsu province, China, were housed in environmentally controlled conditions $\left(22^{\circ} \mathrm{C}\right.$, a $12 \mathrm{~h}$ light/dark cycle with the light cycle from 6:00 to 18:00 and the dark cycle from 18:00 to 6:00) with ad libitum access to standard laboratory chow and water. The study protocol was approved by the local institution review boards and the animal study was carried out in accordance the established ethical guidelines for animal use and care at Nanjing University. C57BL/6 mice were inoculated subcutaneously on the mid-right flank with $5 \times 10^{5} \mathrm{~B} 16 \mathrm{~F} 10$ cells in $0.1 \mathrm{~mL}$ PBS and, at d 6 (or 8) post tumor inoculation, were treated intraperitoneally, intravenously or by direct local injection with $S$. Typhimurium strain VNP20009 at a dose of $10^{5}, 5 \times 10^{6}$ or $5 \times 10^{4}$ CFUs per mouse, respectively. Additionally, mice were treated intraperitoneally with triptolide at $20 \mu \mathrm{g} / \mathrm{kg}$ body per 
mouse from d 4 to $\mathrm{d} 14$ post tumor inoculations. For the neutrophil-depletion experiment, mice were injected intraperitoneally with anti-Gr-1 monoclonal antibody (BD Biosciences, San Diego, CA) or non-immune mouse IgG (R \& D Systems, Minneapolis $\mathrm{MN})$ at $50 \mu \mathrm{g}$ per mouse on $\mathrm{d} 6$ and 8 post tumor inoculations. For the neutralization of VEGF in tumors, anti-VEGF neutralizing antibody ( $R$ \& D Systems) or non-immune mouse IgG was directly injected into tumors at various locations at $10 \mu \mathrm{g}$ per tumor on $\mathrm{d}$ 7, 9 and 10 post tumor inoculations. Tumor volume was determined as previously described [4] by using the formula: tumor volume $=$ length $\times$ width $^{2} \times 0.52$. Survival information was also recorded.

\section{Flow-cytometric analysis}

Tumor mass was digested with collagenase I (Invitrogen, Carlsbad, CA), collagenase IV (Sigma, St. Louis, MO), DNase I (Sigma) and hyaluronidase (Worthington Biochemical Corp, Lakewood, CA ) for $1 \mathrm{~h}$ at $37^{\circ} \mathrm{C}$ for the preparation of the single cell suspension. The cells were blocked and stained with anti-CD4-PE, anti-CD8-FITC, or anti-Gr-1-FITC antibodies (BD Biosciences), and were subjected to flow-cytometry as described [26].

\section{Immunoblotting and RT-PCR assays}

Cellular extracts of B16F10 melanoma cells or Jurkat cells were prepared in the cell extraction buffer as instructed by the manufacturer (Invitrogen). The Immunoblotting procedures were performed as depicted previously [4] and the following antibodies were used: mouse monoclonal antibodies against NF-kBp65 (Santa Cruz Biotechnology, Santa Cruz, CA), rabbit antibodies against phospho-p65, ІкB and GAPDH (all from Cell Signaling, Beverly, CA). For RT-PCR assays, total cellular RNA was extracted using RNeasy Micro or Mini Kit following the manufacturer's protocol (QIAGEN). cDNA synthesis and RT-PCR were performed by using primers for VEGF and the housekeeping gene glyceraldehyde-3-phosphate dehydrogenase (GAPDH) as described before [4].

\section{Luciferase reporter assays}

Jurkat cells were plated in 24-well plates at $10^{4}$ cells $/ 500 \mu \mathrm{L}$. After $24 \mathrm{~h}$, they were transfected with polyethylenimine and $0.5 \mu \mathrm{g}$ mouse NFkB p65 luciferase reporter plasmid and $0.1 \mu \mathrm{g}$ Renilla luciferase reporter vector. After $24 \mathrm{~h}$, the cells were treated with triptolide $(10 \mathrm{ng} / \mathrm{mL})$ for $6 \mathrm{~h}$ followed by treatment with fresh Salmonella VNP20009 for $15 \mathrm{~min}$. The cells were then prepared for luciferase assays using the Dual-Luciferase system (Promega, Madison, WI). Data was expressed as relative luciferase activity representing the mean \pm S.D. of duplicate experiments and were obtained by calculating the ratio of Firefly luciferase activity and Renilla luciferase activity.

\section{Electrophoretic mobility shift assays (EMSA)}

Nuclear extracts were prepared as described [4] and $10 \mu \mathrm{g}$ nuclear extract was pre-incubated with $2 \mu \mathrm{g}$ poly $\mathrm{dI}-\mathrm{dC}$ on ice for $10 \mathrm{~min}$ before incubation with 0.05 pmol FAM-labeled oligonucleotide in binding buffer containing $10 \mathrm{mM}$ Tris-HCl, ( $\mathrm{pH} 7.5), 1 \mathrm{mM}$ $\mathrm{MgCl}_{2}, 0.5 \mathrm{mM}$ EDTA, $0.5 \mathrm{mM}$ dithiothreitol, $50 \mathrm{mM}$ $\mathrm{NaCl}, 4 \%$ glycerol, and $1 \%$ bovine serum albumin for 45 min. The DNA-protein complexes were resolved by running on a $4.5 \%$ polyacrylamide gel. The sequences of the double-stranded EMSA NFKB (p65) probes were: NFkB-forward (FAM), 5'-FAM-AGTTG AGGGGACTTTCCCAGGC-3', and NFkB-forward (c old), 5'-AGTTGAGGGGACTTTCCCAGGC-3' .

\section{Bio-Plex Multiplex Suspension Array and ELISA}

Tumor tissue was weighed and homogenized for $1 \mathrm{~h}$ on ice in $50 \mathrm{mM}$ HEPES (pH 7.4), $100 \mathrm{mM} \mathrm{NaCl}, 50$ $\mathrm{mM} \mathrm{NaF}, 2 \mathrm{mM}$ EDTA, $1 \%$ Triton-100 and $100 \mu \mathrm{g} / \mathrm{mL}$ phenylmethanesulfonylfluoride (PMSF). Cytokines and chemokines were measured using the Bio-Plex Suspension Array System (Bio-Rad Laboratories, Hercules, CA) according to the manufacturer's protocol. For ELISA, tissue homogenates were subjected to the ELISA procedure as described [4] by using the mouse VEGF ELISA kit (Boster, Wuhan, China).

\section{Immunohistochemistry and fluorescent microscopy}

Tumor tissues were fixed with $4 \%$ formaldehyde, embedded in paraffin and sectioned for hematoxylin and eosin (H\&E) staining. For necrosis determination in tumors, the necrotic area was quantitated by software Image J (NIH, Bethesda, $\mathrm{MD})$. Immuno-stainings were performed on frozen tumor sections with appropriate biotinylated antibodies according to standard histological procedures. Gr-1+ and CD31+ cells and VEGF in tumor tissues were stained, and $\mathrm{CD}^{+} 1^{+}$cells were quantitated as previously described [16]. Macrophages in tumors were determined by anti-F4/80-FITC antibody, and GFP-expressing VNP and TUNEL assay were described previously [4].

\section{Statistical analysis}

Student's $t$ test analysis was carried out on data using the SPSS software to assess statistical significance. Differences between experimental groups were considered to be significant when $P<$ 0.05 . 


\section{Results}

Triptolide and VNP20009 synergistically suppressed melanoma growth in vivo by enlarging the necrotic area caused by VNP20009 colonization

The xenografts of murine B16F10 melanoma, which are fast growing and easy to monitor, are commonly used for the evaluation of cancer therapies [25]. To test whether the combination therapy of triptolide and VNP20009 could act synergistically against the B16F10 melanoma, mice bearing melanoma were treated with triptolide alone, VNP200009 alone, or the combination of both. Both the intraperitoneal and intratumoural administration of triptolide alone or VNP20009 alone showed a moderate suppression of the growth of melanoma in mice, and the combination of triptolide and VNP20009 resulted in significantly better antitumour efficacy (Figure 1A, Figure S1A and S1B). Furthermore, the intraperitoneal administration of both triptolide and VNP20009 remarkably prolonged the survival length of the mice bearing subcutaneous melanoma, resulting in a 40 -day survival rate of $85.7 \%$ for the mice that received the combination therapy, which was significantly better than the $14.3 \%$ 40-day survival rate experienced by mice that received the conventional monotherapy of VNP20009 alone (Figure 1B).

The Hematoxylin and eosin (H\&E) staining of tumour tissues showed that the combination therapy resulted in a sharper increase in the number of necrotic tumour cells than with the treatments consisting of either triptolide alone or VNP200009 alone (Figure 1C). Fewer viable tumour cells and a larger area of necrosis were observed in the centre of melanoma tissue from the mice receiving the combination therapy than in the other mice (Figure 1D), suggesting that the synergistic tumour inhibition effects of the combined regimen could be partially attributed to the enhanced necrosis of melanoma cells.

Colony formation assays of bacteria recovered from the tumour and liver tissues demonstrated that both the exact number of tumour-colonized bacteria and the relative tumour specificity of VNP20009 were improved by being combined with triptolide (Figure 1E and 1F). Fluorescent microscopy using VNP20009-expressing green fluorescent protein (VNP-peGFP) further demonstrated that a greater number of VNP-peGFP was localized to the necrotic portion of tumours in the VNP-peGFP-inoculated mice treated with triptolide (Figure 1G).
Triptolide amplified the antitumour effect of VNP20009 by inhibiting the tumour infiltration of inflammatory cells caused by VNP20009 infection

Salmonella-colonized tumours are always infiltrated by various inflammatory cells for the elimination of invading pathogens [26]. The fluorescent immunostaining of F4/80 (a macrophages marker) and the Immunohistochemistry (IHC) staining of Gr-1 (a neutrophils marker) showed that tumours infected with VNP20009 attracted numerous macrophages and neutrophils, which were weakened by triptolide (Figure 2A). The blockage of neutrophils using either triptolide or a Gr-1-neutralizing antibody significantly enhanced the tumour inhibition effect of VNP20009 (Figure 2B and Figure S2). The IHC staining of tumour tissues from the VNP20009-inoculated mice exhibited numerous neutrophils between the necrotic and viable regions of tumours, which confined VNP20009 inside the necrotic area. By contrast, fewer neutrophils were present in the tumours obtained from mice that received the combination of VNP20009 and triptolide or the Gr-1-neutralizing antibody (Figure 2C). The reduction of $\mathrm{Gr}-1^{+}$cells in tumours from mice receiving the combination therapies was also confirmed by flow cytometry analysis (Figure 2D). Colony formation assays showed that a significantly larger number of colonies were produced by the bacteria retrieved from the tumours of mice that received the combination of VNP20009 and triptolide or the Gr-1 neutralizing antibody (Figure 2E). These combination therapies also led to a dramatic expansion of the necrotic area in tumours treated with VNP20009 (Figure 2F).

When infection by Salmonella occurs, the NF-kB pathway is immediately activated in lymphocytes, leading to the massive secretion of various proinflammatory factors [27]. The cytokine and chemokine profiling of tumour tissues by the multiplex suspension array revealed that, compared with the mock treatment using PBS, VNP20009 significantly upregulated the production of proinflammatory factors, such as IL-6, IL- $1 a$, IL-1 $\beta$, IL-12p70, IL-17, and IL-13, and chemokines, such as G-CSF, GM-CSF, MIP-1 $\alpha$, MIP-1 $\beta, \mathrm{KC}$, and eotaxin, in the tumour microenvironment, which was significantly attenuated by triptolide (Figure 3A-3E). Jurkat cells, an immortalized line of human $\mathrm{T}$ lymphocyte cells, were treated with VNP20009 and triptolide to test their effects on the NF-kB pathway in vitro. Immunoblottings of cell lysates from VNP20009-treated Jurkat cells showed that VNP20009 caused rapid NFKB (p65) phosphorylation, which was strongly suppressed by triptolide (Figure 3F). 
A

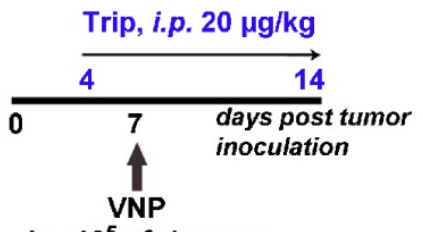

i.p. $10^{5} \mathrm{cfu} /$ mouse

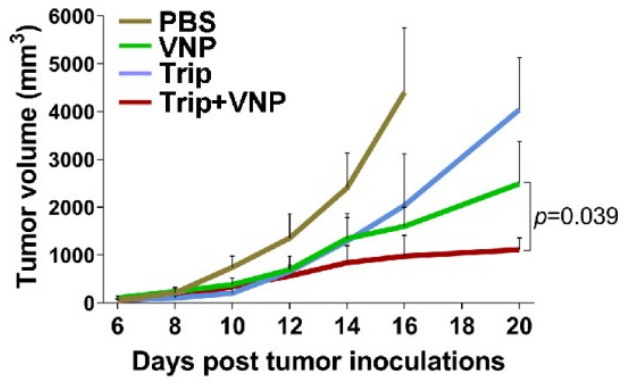

C

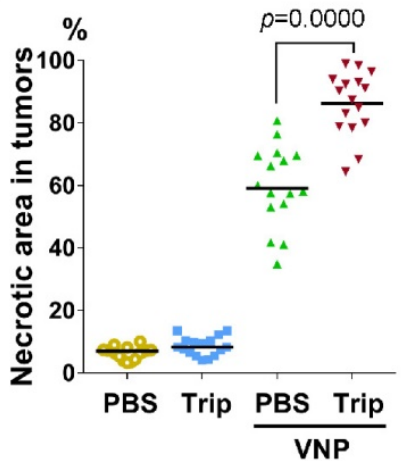

D

B

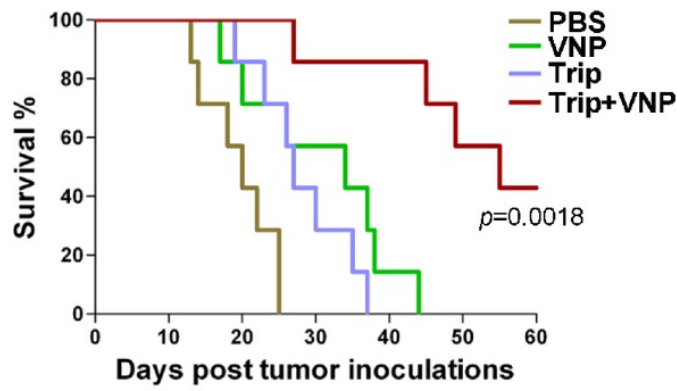

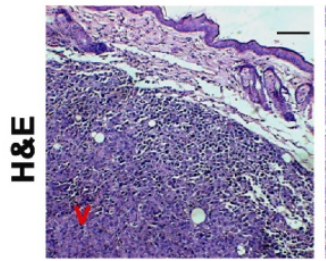

E

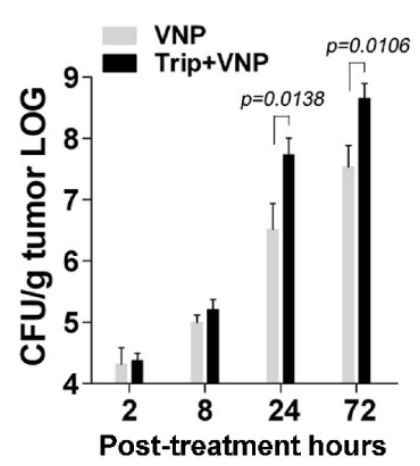

G

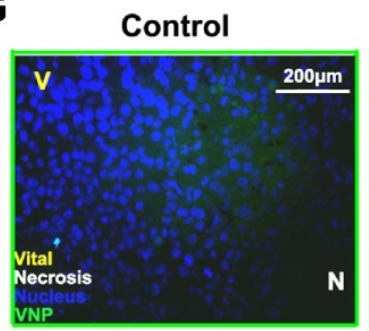

$\mathbf{F}$

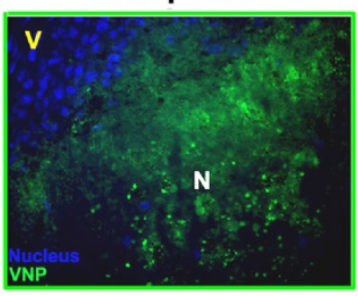

VNP

Trip
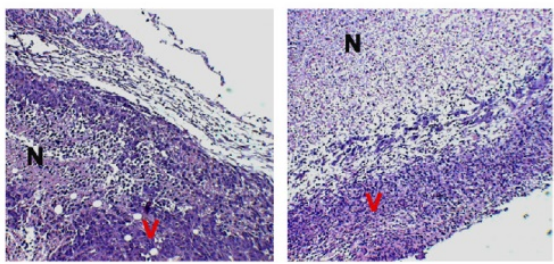

$\mathbf{F}$

\section{Trip+VNP}

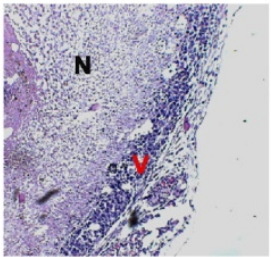

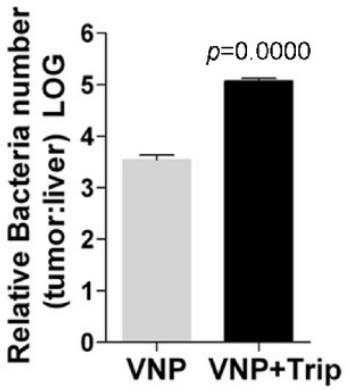

Trip+VNP-peGFP

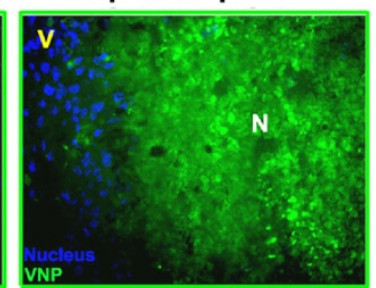

Figure 1. Triptolide Enhances the Anti-tumour Effect of VNP20009 by Enlarging the Necrotic Area Caused by VNP Colonization A) The treatment schedule of triptolide and VNP20009 (VNP) for mice bearing B16F10 melanoma xenografts, and the volume of tumours in different treatment groups. Triptolide was given intraperitoneally once every day from d 4 to d 14 at $20 \mu \mathrm{g} / \mathrm{kg}$ in $200 \mu \mathrm{L} \mathrm{PBS}$. Mean + S.D.. $n=7$. B) The Kaplan-Meier survival curves of mice bearing melanoma xenografts after intraperitoneal administration of triptolide and VNP, $n=7$, $P$ value represents VNP versus the combination of triptolide and VNP. C) The quota of the percentage of necrotic areas in sections of tumours harvested on $d 10$ post treatment. In each section per tumour, four random areas were counted, and four tumours were harvested in each treatment group. The quota of the percentage of necrosis was determined using Image $J$ software. Mean \pm S.D., $n=16$. D) H\&E staining (bar represents $300 \mu \mathrm{m}$ ) of tumours treated with triptolide, VNP, or the combination of triptolide and VNP. N: necrotic areas, V: viable tumour cells. E) The numbers of bacterium colonized in the tumors at $2,8,24$ and $72 \mathrm{~h}$ post infection. Mean \pm S.D., $n=3$, All the assays were repeated three times independently. F) The ratio of bacterial colonization in tumours and livers on $\mathrm{d} 3$ post the intraperitoneal injection of $\mathrm{VNP}$ at $10^{5} \mathrm{CFU} /$ mouse. G) Fluorescent microscopy of melanoma tissues from mice receiving different treatments of triptolide and VNP expressing GFP. The nuclei were stained with DAPI, N: necrotic area, V: viable tumour cells. 
A

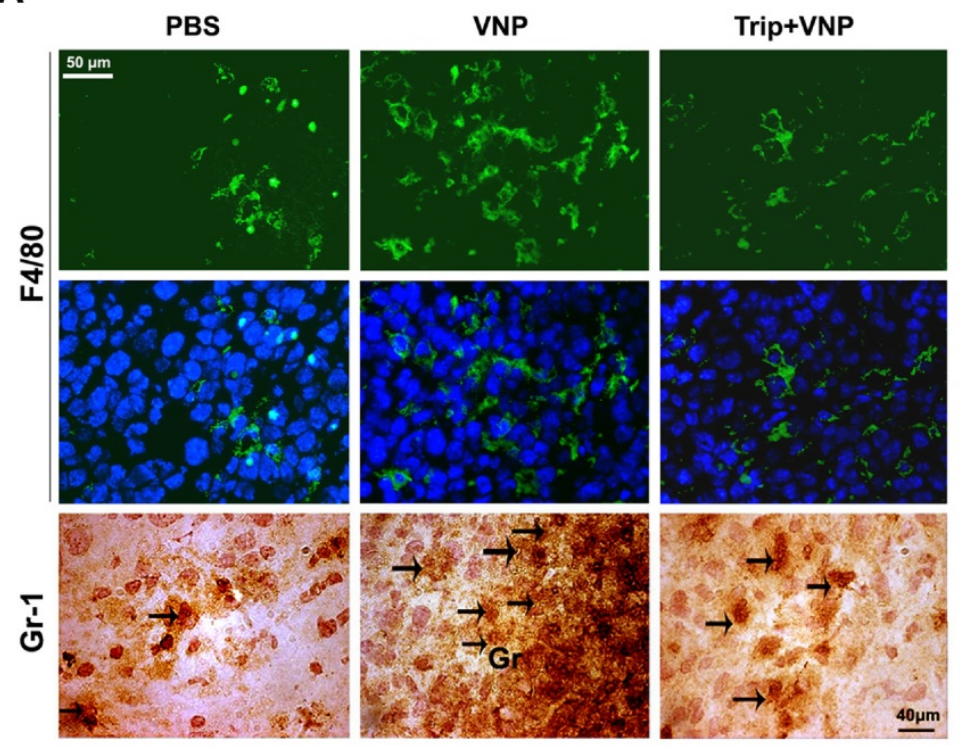

B

Gr-1/lgG antibodies

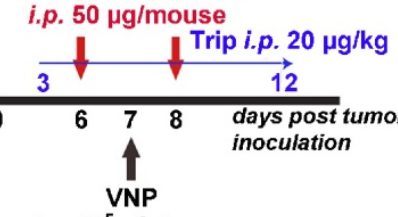

i.p. $10^{5} \mathrm{cfu} /$ mouse

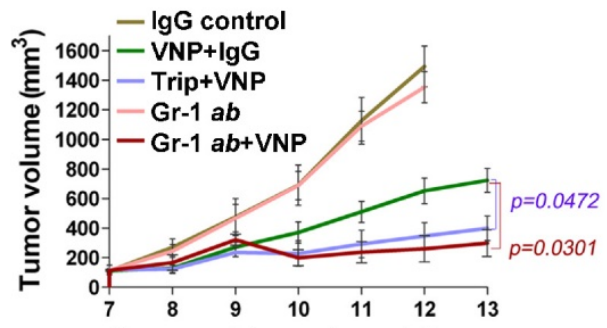

Days post tumor inoculations

C

IgG control

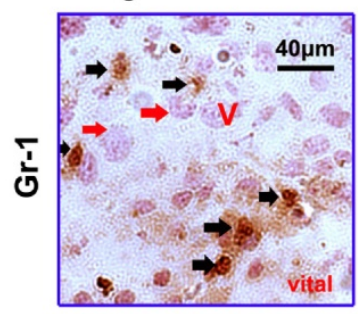

IgG+VNP

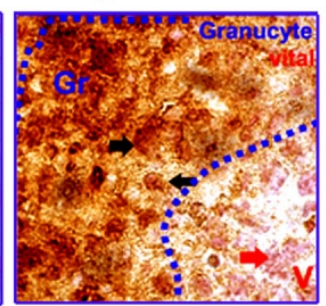

Trip+VNP

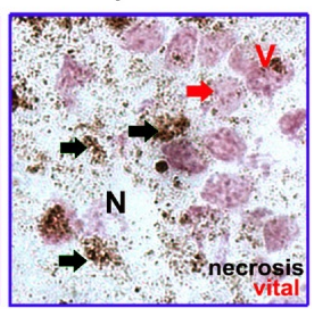

Gr-1+VNP

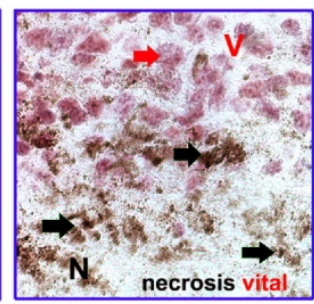

D

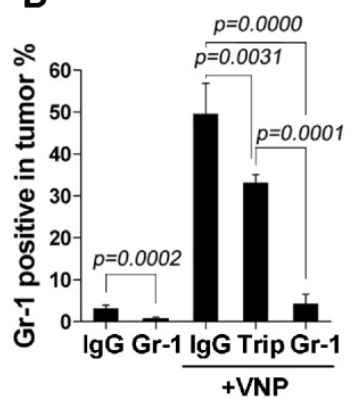

E

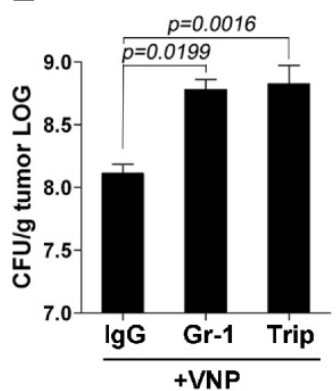

$\mathbf{F}$

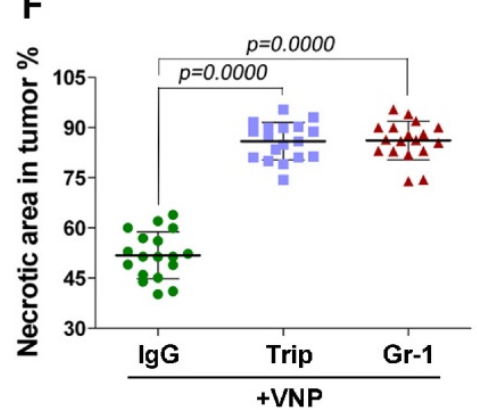

Figure 2. Triptolide Amplifies the Anti-tumour Effect of VNP20009 by Inhibiting the Infiltration of Inflammatory Cells Caused by VNP20009 Infection. A) Fluorescent microscopy of $\mathrm{F} 4 / 80^{+}$macrophages and immunohistochemistry of $\mathrm{Gr}-1^{+}$granulocytes in tumours. The F4/80 antibody was tagged with FITC and the nucleus was stained with DAPI. The Gr-1+ cells are indicated by arrows. B) The treatment schedule of neutrophil-depletion experiment in melanoma bearing mice, and the volume of tumours in different treatment groups. Gr-1 neutralizing antibody or its lgG control $(50 \mu \mathrm{g} /$ mouse) was injected twice intraperitoneally on $\mathrm{d} 6$ and $\mathrm{d} 8$, triptolide ( $20 \mu \mathrm{g} /$ mouse) was injected once daily intraperitoneally from $\mathrm{d} 3$ to $\mathrm{d} 12$ and $10^{5} \mathrm{CFU} /$ mouse VNP20009 (VNP) was intraperitoneally injected on d 7 post tumour-inoculation. Mean \pm S.D., $n=5$. C) IHC stainings of Gr-1 in tumours from the neutrophil- depletion experiment on $\mathrm{d} 7$ post VNP infection. Black arrows indicate Gr-1+ cells. Red arrows indicate tumour cells. D) Intratumoral Gr-1+ cells were measured by the flow-cytometry on $\mathrm{d} 7$ post VNP infection. Mean \pm S.D., $n=3$. E) Relative tumour bacterial colonization on $d 7$ post VNP infection. Mean \pm S.D., $n=3$. F) The quantification of necrotic area in tumours from the neutrophil-depleted mice. Three tumours were harvested in each group. Six parts at random locations were fixed and sectioned in each tumour, and the percentage of necrotic was calculated by Image J software. Mean \pm S.D., $n=16$. 
A

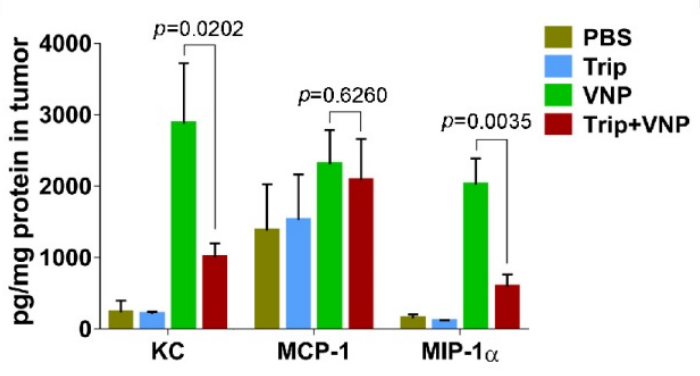

C

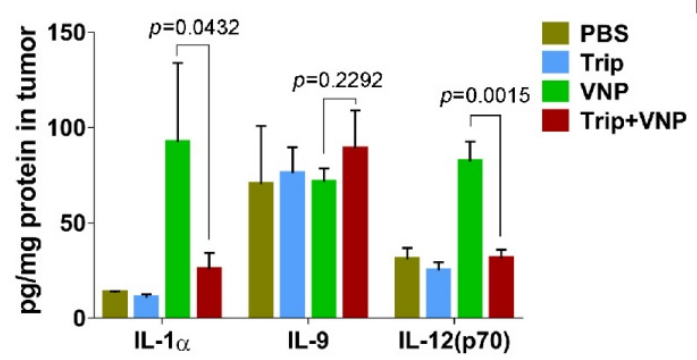

E

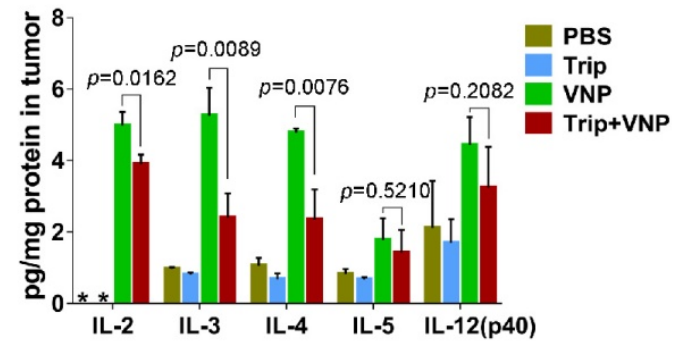

B

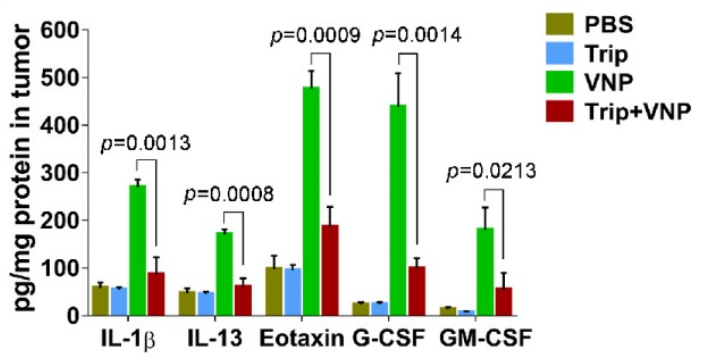

D

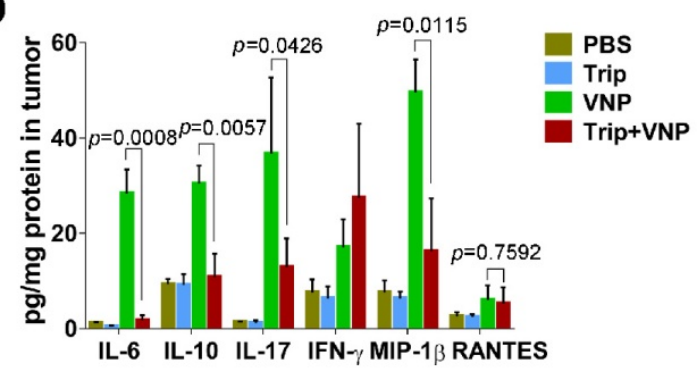

$\mathbf{F}$

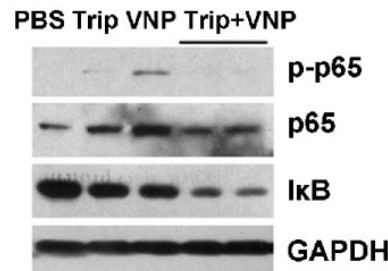

G

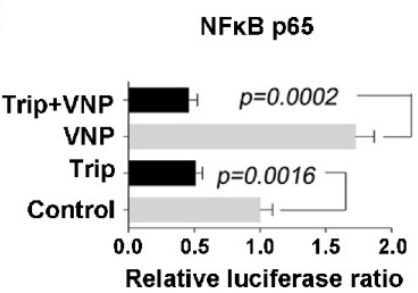

$\mathrm{H}$

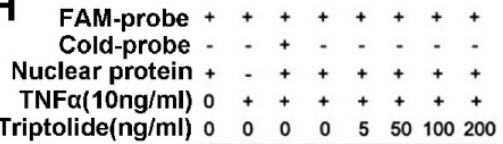

Triptolide(ng/ml) $0 \quad 0 \quad 0 \quad 0 \quad 5 \quad 50100200$

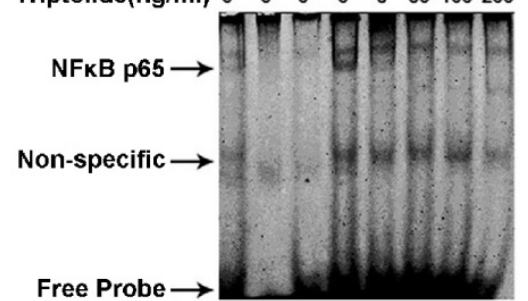

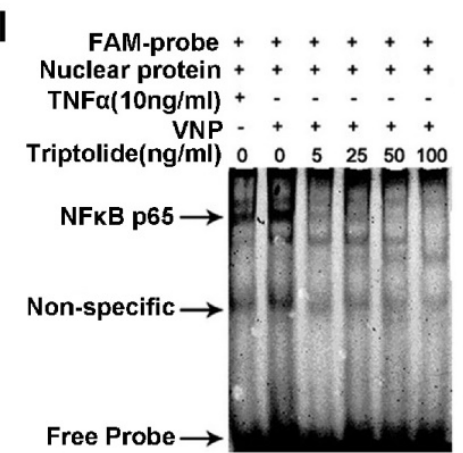

Figure 3. Triptolide Inhibits the Production of Cytokines and Chemokines by Suppressing the NFkB Pathway of the Tumour-related Inflammatory Cells. A-E) The Bio-plex Multiplex Suspension Array of cytokines in tumours, Mean \pm S.D., $n=3$, * below detection limits. F) Immunoblotting assays showed that triptolide inhibited the NFkBp65 phosphorylation induced by VNP infection in Jurkat cells. G) NFkBp65 luciferase reporter assays showed that triptolide inhibited the transcriptional activity of NFkBp65 which was activated by VNP infection in Jurkat cells. The mean value of the control group was defined as 1.0. Mean \pm S.D., $n=3$. H) The EMSA assay showed that triptolide exhibited a dose-dependent inhibition on the TNF- $\alpha$-induced DNA binding activity of NFKBp65 in Jurkat cells. I) The EMSA assay showed that triptolide exhibited a dose-dependent inhibition on the VNP-induced DNA binding activity of NFKBp65 in Jurkat cells.

Luciferase reporter assays further showed that VNP20009 rapidly induced the transcriptional activity of NFKB (p65), which could be significantly inhibited by triptolide (Figure 3G). Moreover, electrophoretic mobility shift assays demonstrated that VNP20009 enhanced the DNA binding activity of p65, while triptolide significantly attenuated the TNF- $\mathrm{a}-$ or VNP20009-induced DNA binding activity of p65 in Jurkat cells (Figure $\mathbf{3 H}$ and 3I). The results of these experiments suggest that the activation of lymphocytes by VNP20009 is weakened by triptolide, which is consistent with previous reports about triptolide's immunosuppressive functions [28, 29].

Our data shows that by suppressing the tumour infiltration of neutrophils and by downregulating the production of proinflammatory cytokines and chemokines, triptolide improves the survival and growth of VNP20009 inside melanoma tissues, leading to enhanced tumour necrosis. 


\section{Triptolide and VNP20009 exerted antineoangiogenesis effects by reducing CD31+ cells and inhibiting VEGF expression in melanoma}

Angiogenesis is critical to tumour growth, metastasis, and neoplastic progression [30]. A strong staining of CD31, an important neovascularization marker, indicates active angiogenesis [31], which could improve the influx of oxygen inside the tumour. However, a hypoxic tumour microenvironment is more conducive to the proliferation of Salmonella [14].

Triptolide has been characterized as a compound that suppresses angiogenesis [22], and there have been some successful instances of improving Salmonella therapy with antiangiogenesis drugs [17]. In this study, we were interested in discovering whether triptolide could exert synergistic antiangiogenesis effects when used with VNP20009 to achieve a better inhibitory effect on tumour growth in melanoma-bearing mice. The IHC staining of CD31 showed that triptolide by itself reduced the number of CD31 positive cells in melanoma, which was further accentuated by VNP20009 (Figure 4A and 4B). Cancer cells secrete VEGF to induce angiogenesis inside the tumour to support the tumour's growth [35], so a high VEGF protein level is strongly correlated with poor prognosis [36]. The IHC staining of VEGF in melanoma tissue, the RT-PCR analysis of the RNA level of VEGF in melanoma tissue, and the ELISA assay's measurement of the VEGF level in the lysates of melanoma tissue all showed that triptolide and VNP20009 synergistically inhibited VEGF expression in melanoma tissue (Figure 4A, 4C, and 4D), suggesting that triptolide and VNP20009 could strongly inhibit tumour angiogenesis in a synergistic manner.

To test whether suppressing tumour angiogenesis could directly improve the antitumour effects of VNP20009, VEGF was specifically depleted by a neutralizing antibody in the VNP20009-inoculated mice, and the growth of tumour was closely monitored. The tumour growth was more significantly slowed by the combination therapy of the VEGF-neutralizing antibody and VNP20009 than with monotherapies consisting of the VEGF-neutralizing antibody alone or VNP20009 alone (Figure 4E and Figure S3). At the same time, a better colonization of VNP20009 was observed in the tumours of mice receiving the combination of VNP20009 and the VEGF-neutralizing antibody than in the tumours of mice receiving the combination of VNP20009 and the nonspecific antibody (Figure 4F), indicating that VEGF depletion directly improved VNP20009 therapy by enhancing the tumour colonization of the bacteria. Triptolide, which suppressed the expression of VEGF, could contribute to VNP20009 therapy partially through its antiangiogenesis activity.

In summary, our study showed that the combination therapy of triptolide and VNP20009 exhibited significantly improved efficacy compared with the conventional monotherapy of VNP20009 alone. Triptolide enhanced the tumour colonization of VNP20009 by suppressing the angiogenesis and the infiltration of inflammatory cells in the tumours, which were two important factors limiting the antitumour effects of highly attenuated VNP20009 (Figure S4).

\section{Discussion}

As a pathogen that causes typhoid fever, Salmonella has a dramatic influence on the immune system, quickly activating multiple host immune responses to fight the pathogen. A variety of bone marrow-derived phagocytes, including neutrophils, macrophages, and natural killer cells are recruited to the site of infection to phagocytose bacteria and to present the antigen to initiate an adaptive immune response [34]. During Salmonella-mediated cancer therapy, such a process occurs, but it occurs in a comparatively milder manner due to the use of the attenuated strains. In this case, the host immune response, which tries to clear Salmonella from the body, is an important factor that limits the efficacy of the Salmonella-mediated cancer therapy-this response is a double-edged sword [11-13].

Tumour-targeting VNP20009 attracts various inflammatory cells, such as neutrophils, macrophages, and $\mathrm{CD}^{+} \mathrm{T}$ cells to a tumour [11-13]. Neutrophils and macrophages suppress VNP20009 via their bactericidal activities [11], and $\mathrm{CD}^{+} \mathrm{T}$ cells can eliminate tumour cells by releasing cytotoxic granules $[12,13]$. However, $\mathrm{CD}^{+}$cytotoxic $\mathrm{T}$ cells are often dysfunctional in the tumour microenvironment due to the upregulation of Programmed cell death protein 1 (PD-1) and abnormally active regulatory $\mathrm{T}$ cells, which create an immunosuppressive microenvironment in the tumour $[35,36]$. Salmonella infection can break this immunosuppressive status by strongly activating the $\mathrm{NFKB}$ pathway in various lymphocytes [27]. Salmonella can reportedly promote the tumour infiltration of $\mathrm{CD}^{+} \mathrm{T}$ cells [37]. In this study, we also found that a low dose of triptolide (20 $\mu \mathrm{g} / \mathrm{kg}$ ) promoted the VNP20009-induced tumour infiltration of $\mathrm{CD}^{+} \mathrm{T}$ cells (Figure S5A and S5B), while a high dose of triptolide $(\geq 100 \mu \mathrm{g} / \mathrm{kg})$ exhibited a broad-spectrum suppressive effect on immunity, resulting in the loss of triptolide's synergistic antitumour effect (Figure S6A-S6E). 
A
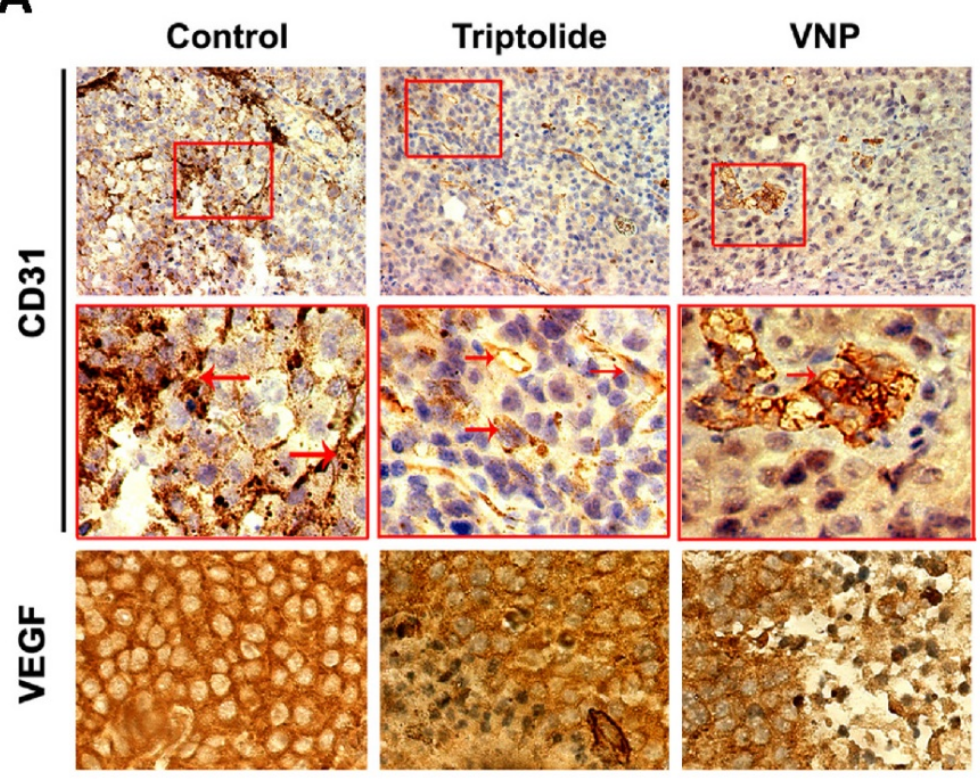

VNP+Triptolide

B

CD31

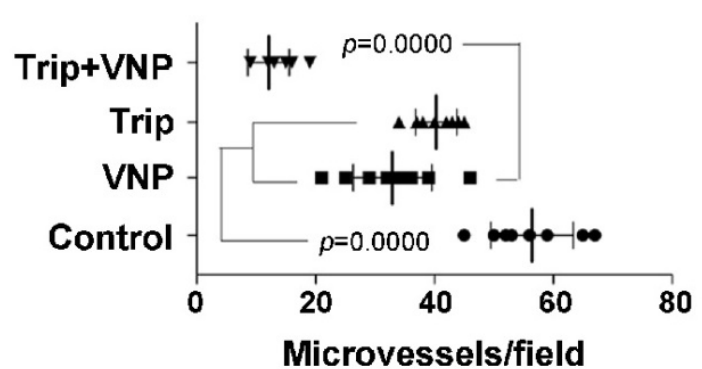

E $\quad$ VEGF ab i.t.

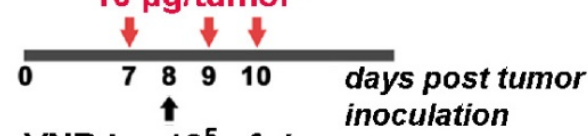

VNP i.p. $10^{5} \mathrm{cfu} / \mathrm{mouse}$

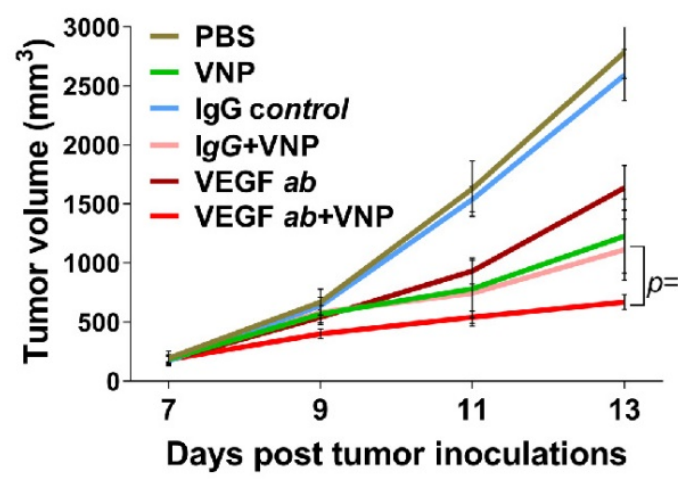

C
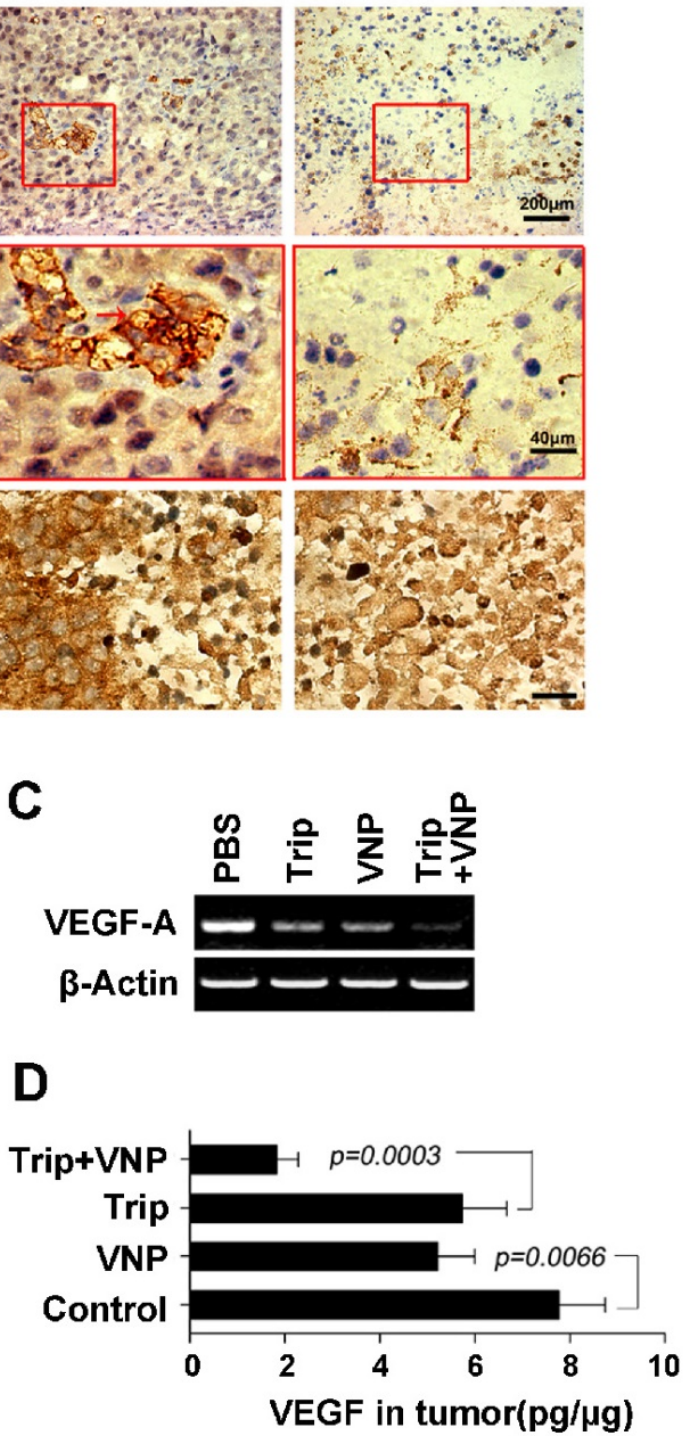

$\mathbf{F}$

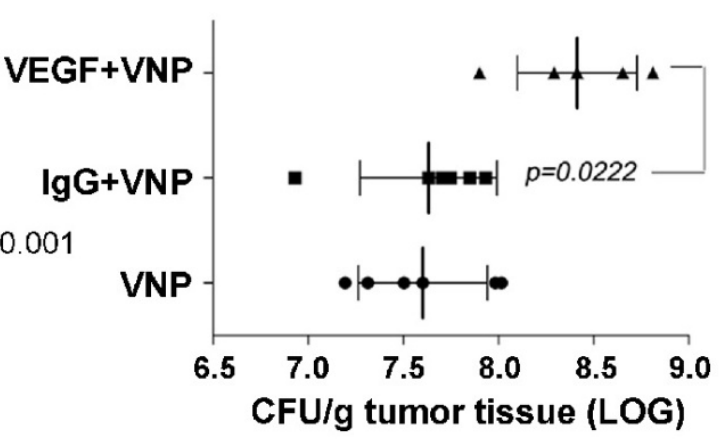

Figure 4. Triptolide and VNP20009 Synergistically Inhibit the Tumour Angiogenisis by Suppressing the Production of VEGF. A) Immunohistochemistry of CD31+ cells and VEGF expression in melanoma tumours from mice receiving triptolide and/or VNP20009 (VNP) treatments on d 7 post infection. CD31+ cells are indicated by red arrows. Bars on VEGF sections represent $10 \mu \mathrm{m}$. B) The quantification of CD31+ microvessels in tumours. Two sections per tumour were determined and four tumours were harvested in each group. The number of the CD31+ cells was calculated. Mean \pm S.D., $n=8$. C) VEGF expression in melanoma tumours was examined by RT-PCR. D) ELISA assays were performed to determine VEGF concentration in tumours. Mean \pm S.D., $n=4$. E) The treatment schedule of the combination therapy of VNP and VEGF neutralizing antibody, and its effect on the growth of tumours. The VEGF neutralizing antibody ( $10 \mu g /$ tumour) or the IgG control antibody ( $10 \mu \mathrm{g} /$ tumour) were injected directly into the tumours three times on $\mathrm{d} 7,9$ and 10 . VNP was intraperitoneally injected on $\mathrm{d} 8$ post tumour inoculation. Mean \pm S.D., $n=5$. F) The bacterial colonization in the tumours was determined on $d 5$ post VNP infection in the VEGF neutralization assay. Mean \pm S.D., $n=5$. 
The antitumour effect of VNP20009 was weaker in immunodeficient mice than in mice with an intact immune system [8]. However, another strain of $S$. Typhimurium, A1-R, which was specially isolated from A1-infected tumours in immunodeficient mice, showed strong antitumour effects in several kinds of PDOX immunodeficient mouse models, including pancreatic cancer, cervical cancer, soft-tissue sarcoma, and breast cancer [38-40]. Apart from suppressing the growth of primary tumours, A1-R could also reduce brain and bone metastasis in experimental breast cancer metastatic mouse models [41, 42].

These observations indicate that host immunity is actively involved during the process of Salmonella-mediated cancer therapy even though the extent varies with strains. In the necrotic area, the lysis of tumour cells provides a large pool of tumour-specific antigens, which could trigger an adaptive immune response against tumour cells by the infiltrated phagocytes [26]. As a strong stimulus for the host immune response and as a tumour-specific vehicle, Salmonella has been genetically engineered as a carrier of tumour-specific antigens for the cancer immune therapy, achieving complete tumour regression and the generation of a lifelong protective memory in an A20 lymphoma model [43].

Therefore, the careful manipulation of the host immunity, such as the suppression of $\mathrm{Gr}-1^{+}$ neutrophils and the activation of $\mathrm{CD}^{+} \mathrm{T}$ cells using either a low dose of triptolide or other compounds, can potentiate the colonization of VNP20009 inside tumours to maximize VNP20009's antitumour effect. At the same time, further studies on the Salmonella-mediated tumour therapy can provide important knowledge about the crosstalk between cancer and host immunity to promote the development of vaccinations against cancer.

\section{Supplementary Material}

Supplementary figures.

http://www.thno.org/v07p2250s1.pdf

\section{Acknowledgements}

This study was supported in part by grants from the Doctoral Station Science Foundation by the Chinese Ministry of Education (20130091130003), the Chinese National Natural Sciences Foundation (81630092, 81573338, 81421091), the National Key Research Program by Ministry of Science and Technology (2014CB744501, 2016YFC0902700).

\section{Competing Interests}

The authors have declared that no competing interest exists.

\section{References}

1. Pawelek JM, Low KB, Bermudes D. Tumor-targeted Salmonella as a novel anticancer vector. Cancer Res. 1997; 57: 4537-44.

2. Morrissey D, O'Sullivan GC, Tangney M. Tumour targeting with systemically administered bacteria. Curr Gene Ther. 2010; 10: 3-14

3. Low KB, Ittensohn M, Le T, Platt J, Sodi S, Amoss M, et al. Lipid A mutant Salmonella with suppressed virulence and TNFalpha induction retain tumor-targeting in vivo. Nat Biotechnol. 1999; 17: 37-41.

4. Chen J, Wei D, Zhuang H, Qiao Y, Tang B, Zhang X, et al. Proteomic screening of anaerobically regulated promoters from Salmonella and its antitumor applications. Mol Cell Proteomics. 2011; 10: M111 009399.

5. Toso JF, Gill VI, Hwu P, Marincola FM, Restifo NP, Schwartzentruber DJ, et al. Phase I study of the intravenous administration of attenuated Salmonella typhimurium to patients with metastatic melanoma. J Clin Oncol. 2002; 20: $142-52$

6. Nemunaitis J, Cunningham C, Senzer N, Kuhn J, Cramm J, Litz C, et al. Pilot trial of genetically modified, attenuated Salmonella expressing the E. coli cytosine deaminase gene in refractory cancer patients. Cancer Gene Ther. 2003; 10: 737-44

7. Lee $\mathrm{CH}, \mathrm{Wu} \mathrm{CL}$, Chen $\mathrm{SH}$, Shiau AL. Humoral immune responses inhibit the antitumor activities mediated by Salmonella enterica serovar choleraesuis. J Immunother. 2009; 32: 376-88.

8. Lee CH, Hsieh JL, Wu CL, Hsu PY, Shiau AL. T cell augments the antitumor activity of tumor-targeting Salmonella. Appl Microbiol Biotechnol. 2011; 90: 1381-8.

9. Lee $\mathrm{CH}, \mathrm{Hsieh} \mathrm{JL}, \mathrm{Wu} \mathrm{CL}, \mathrm{Hsu} \mathrm{HC}$, Shiau AL. B cells are required for tumor-targeting Salmonella in host. Appl Microbiol Biotechnol. 2011; 92: 1251-60.

10. Saltzman DA, Katsanis E, Heise CP, Hasz DE, Vigdorovich V, Kelly SM, et al. Antitumor mechanisms of attenuated Salmonella typhimurium containing the gene for human interleukin-2: a novel antitumor agent? J Pediatr Surg. 1997; 32: 301-6.

11. Westphal K, Leschner S, Jablonska J, Loessner H, Weiss S. Containment of tumor-colonizing bacteria by host neutrophils. Cancer Res. 2008; 68: 2952-60.

12. Domingos-Pereira S, Hojeij R, Reggi E, Derre L, Chevalier MF, Romero P, et al. Local Salmonella immunostimulation recruits vaccine-specific CD8 T cells and increases regression of bladder tumor. Oncoimmunology. 2015; 4: e1016697.

13. Roider E, Jellbauer S, Kohn B, Berchtold C, Partilla M, Busch DH, et al. Invasion and destruction of a murine fibrosarcoma by Salmonella-induced effector CD8 T cells as a therapeutic intervention against cancer. Cancer Immunol Immunother. 2011; 60: 371-80.

14. Wall DM, Srikanth CV, McCormick BA. Targeting tumors with salmonella Typhimurium- potential for therapy. Oncotarget. 2010; 1: 721-8.

15. Zhao M, Geller J, Ma H, Yang M, Penman S, Hoffman RM. Monotherapy with a tumor-targeting mutant of Salmonella typhimurium cures orthotopic metastatic mouse models of human prostate cancer. Proc Natl Acad Sci U S A. 2007: 104: 10170-4.

16. Jia LJ, Wei DP, Sun QM, Jin GH, Li SF, Huang Y, et al. Tumor-targeting Salmonella typhimurium improves cyclophosphamide chemotherapy at maximum tolerated dose and low-dose metronomic regimens in a murine melanoma model. Int J Cancer. 2007; 121: 666-74

17. Hiroshima Y, Zhang Y, Murakami T, Maawy A, Miwa S, Yamamoto M, et al. Efficacy of tumor-targeting Salmonella typhimurium A1-R in combination with anti-angiogenesis therapy on a pancreatic cancer patient-derived orthotopic xenograft (PDOX) and cell line mouse models. Oncotarget. 2014; 5: 12346-57.

18. Kupchan SM, Court WA, Dailey RG, Jr., Gilmore CJ, Bryan RF. Triptolide and tripdiolide, novel antileukemic diterpenoid triepoxides from Tripterygium wilfordii. J Am Chem Soc. 1972; 94: 7194-5.

19. Yang S, Chen J, Guo Z, Xu XM, Wang L, Pei XF, et al. Triptolide inhibits the growth and metastasis of solid tumors. Mol Cancer Ther. 2003; 2: 65-72.

20. Wang C, Liu B, Xu X, Zhuang B, Li H, Yin J, et al. Toward targeted therapy in chemotherapy-resistant pancreatic cancer with a smart triptolide nanomedicine. Oncotarget. 2016; 7: 8360-72

21. Jiang QW, Cheng KJ, Mei XL, Qiu JG, Zhang WJ, Xue YQ, et al. Synergistic anticancer effects of triptolide and celastrol, two main compounds from thunder god vine Oncotarget. 2015: 6: 32790-804

22. He MF, Huang YH, Wu LW, Ge W, Shaw PC, But PP. Triptolide functions as a potent angiogenesis inhibitor. Int J Cancer. 2010; 126: 266-78.

23. Wang $G$, Wang $X, X u \quad X$. Triptolide potentiates lung cancer cells to cisplatin-induced apoptosis by selectively inhibiting the NER activity. Biomark Res. 2015; 3: 17.

24. Cunningham C, Nemunaitis J. A phase I trial of genetically modified Salmonella typhimurium expressing cytosine deaminase (TAPET-CD, VNP20029) administered by intratumoral injection in combination with 5-fluorocytosine for patients with advanced or metastatic cancer. Protocol no: CL-017. Version: April 9, 2001. Hum Gene Ther. 2001; 12: 1594-6.

25. Overwijk WW, Restifo NP. B16 as a mouse model for human melanoma. Curr Protoc Immunol. 2001; Chapter 20: Unit 20.1.

26. Avogadri F, Martinoli C, Petrovska L, Chiodoni C, Transidico P, Bronte V, et al. Cancer immunotherapy based on killing of Salmonella-infected tumor cells. Cancer Res. 2005; 65: 3920-7.

27. Johannessen M, Askarian F, Sangvik M, Sollid JE. Bacterial interference with canonical NFkappaB signalling. Microbiology. 2013; 159: 2001-13. 
28. Qiu D, Kao PN. Immunosuppressive and anti-inflammatory mechanisms of triptolide, the principal active diterpenoid from the Chinese medicinal herb Tripterygium wilfordii Hook. f. Drugs R D. 2003; 4: 1-18.

29. Krakauer $T$, Chen $X$, Howard OM, Young HA. Triptolide attenuates endotoxin- and staphylococcal exotoxin-induced T-cell proliferation and production of cytokines and chemokines. Immunopharmacol Immunotoxicol. 2005; 27: 53-66.

30. Meert AP, Paesmans M, Martin B, Delmotte P, Berghmans T, Verdebout JM, et al. The role of microvessel density on the survival of patients with lung cancer: a systematic review of the literature with meta-analysis. Br J Cancer. 2002; 87: 694-701.

31. Wang D, Stockard CR, Harkins L, Lott P, Salih C, Yuan K, et al. Immunohistochemistry in the evaluation of neovascularization in tumor xenografts. Biotech Histochem. 2008; 83: 179-89.

32. Carmeliet P. VEGF as a key mediator of angiogenesis in cancer. Oncology. 2005; 69 (Suppl 3): 4-10.

33. Lee JC, Chow NH, Wang ST, Huang SM. Prognostic value of vascular endothelial growth factor expression in colorectal cancer patients. Eur J Cancer. 2000; 36: 748-53.

34. Pham $\mathrm{OH}, \mathrm{McSorley}$ SJ. Protective host immune responses to Salmonella infection. Future Microbiol. 2015; 10: 101-10.

35. Ahmadzadeh M, Johnson LA, Heemskerk B, Wunderlich JR, Dudley ME, White DE, et al. Tumor antigen-specific CD8 T cells infiltrating the tumor express high levels of PD-1 and are functionally impaired. Blood. 2009; 114: 1537-44.

36. Chen ML, Pittet MJ, Gorelik L, Flavell RA, Weissleder R, von Boehmer H, et al. Regulatory $\mathrm{T}$ cells suppress tumor-specific CD8 $\mathrm{T}$ cell cytotoxicity through TGF-beta signals in vivo. Proc Natl Acad Sci U S A. 2005; 102: 419-24.

37. Lee $\mathrm{CH}, \mathrm{Wu} \mathrm{CL}$, Shiau AL. Salmonella choleraesuis as an anticancer agent in a syngeneic model of orthotopic hepatocellular carcinoma. Int J Cancer. 2008; 122: $930-5$.

38. Yano S, Zhang Y, Zhao M, Hiroshima Y, Miwa S, Uehara F, et al. Tumor-targeting Salmonella typhimurium A1-R decoys quiescent cancer cells to cycle as visualized by FUCCI imaging and become sensitive to chemotherapy. Cell cycle. 2014; 13: 3958-63.

39. Hiroshima Y, Zhao M, Zhang Y, Zhang N, Maawy A, Murakami T, et al. Tumor-Targeting Salmonella typhimurium A1-R Arrests a Chemo-Resistant Patient Soft-Tissue Sarcoma in Nude Mice. PloS one. 2015; 10: e0134324.

40. Murakami T, Igarashi K, Kawaguchi K, Kiyuna T, Zhang Y, Zhao M, et al. Tumor-targeting Salmonella typhimurium A1-R regresses an osteosarcoma in a patient-derived xenograft model resistant to a molecular-targeting drug. Oncotarget. 2016.

41. Miwa S, Yano S, Zhang Y, Matsumoto Y, Uehara F, Yamamoto M, et al. Tumor-targeting Salmonella typhimurium A1-R prevents experimental human breast cancer bone metastasis in nude mice. Oncotarget. 2014; 5: 7119-25.

42. Zhang Y, Miwa S, Zhang N, Hoffman RM, Zhao M. Tumor-targeting Salmonella typhimurium A1-R arrests growth of breast-cancer brain metastasis. Oncotarget. 2015; 6: 2615-22.

43. Xu X, Hegazy WA, Guo L, Gao X, Courtney AN, Kurbanov S, et al. Effective cancer vaccine platform based on attenuated salmonella and a type III secretion system. Cancer Res. 2014; 74: 6260-70. 